\section{Should Entry-to-Practice Doctor of Pharmacy (PharmD) Curricula Have at Least One Mandatory Experiential Internship Focusing on Cultural Awareness Training?}

\section{THE "PRO" SIDE}

Cross-cultural training is essential for development of the knowledge, skills, and attitudes necessary for pharmacists to provide competent care within culturally diverse interactions. ${ }^{1}$ Culture, broadly defined as the values and beliefs held by a specific group of people, is influenced by factors such as ethnicity, language, gender, socioeconomic status, and sexual orientation. ${ }^{2}$ In situations of diversity, it is known that health care provider factors (bias, stereotyping, uncertainty) may result in health disparities and suboptimal patient outcomes. ${ }^{3}$ Exercises in cultural awareness, cultural sensitivity, and cross-cultural communication may promote providers' awareness of their own cultural biases and lead to more effective patient care.

Education and practice standards require that pharmacists provide care in culturally diverse environments. The educational outcomes of the Association of Faculties of Pharmacy of Canada (AFPC) explicitly state that pharmacy graduates should be able to "integrate patient preferences related to culture, beliefs and practices" and "demonstrate sensitivity, respect and empathy in intercultural and inter-professional situations". ${ }^{4}$ Additionally, the National Association of Pharmacy Regulatory Authorities (NAPRA) states in its professional competencies that new graduates should be able to assess the patient's health and drug therapy needs, "considering the impact of factors such as culture, language, demographic and physical characteristics". ${ }^{5}$ These standards support the need for curricula designed to promote cultural awareness for pharmacy students graduating from accredited Canadian universities.

A large amount of literature exists regarding cultural competency and the methods used to teach this topic and assess students. ${ }^{3,6}$ In the past, checklists and rubrics aimed at ensuring that students asked certain questions or provided specific recommendations were used to guide the determination of cultural competency levels. Current movements in teaching and assessment recommend against this approach, as student selfreflection and awareness are rarely achieved. Instead, facilitated and self-reflection, along with direct performance feedback, are purported to be higher-level strategies to enhance learning and associated behavioural change. ${ }^{7}$ Therefore, the learning environment and assessment context are important variables to consider when designing curricula.

The classroom setting is typically a suboptimal context in which to teach and evaluate competencies related to cultural sensitivity, awareness, and cross-cultural communication. This unsuitability is largely due to the highly individualized nature of culturally diverse interactions, unclear definitions of culture, and poor predictability of student responses to interactions deemed to be culturally diverse. In addition, a systematic review identified common assumptions about educational methods to teach and assess competent practice across cultural and social diversity. ${ }^{8}$ These assumptions criticized the current educational practices of cultural competency and should be considered when designing new teaching and assessment methods. The systematic review highlighted, among others, the assumptions that culture is a matter of ethnicity, that culture is always possessed by the "other", that the problem of cultural incompetence lies in the practitioner's discriminatory attitudes, and that cross-cultural care is primarily based on white practitioners working with patients from ethnic minority groups. Cases and examples developed by educators are likely to reflect these assumptions, as this is primarily how cultural competency has been traditionally approached. ${ }^{8}$ Alternatively, educators can introduce concepts of providing culturally diverse care in the classroom and then rely on experiential training to reinforce and further develop students' attitudes and skills. A casebased, "one size fits all" approach is not realistic, as it is impossible to predict when students (or practitioners) will find themselves in situations of cultural conflict.

Experiential internships as an educational context for development of cultural awareness and cultural sensitivity overcome the limitations mentioned above. Cultural training must be tailored to the individual's specific learning needs, as it is unknown when that person might encounter a cultural moment. ${ }^{9}$ Experiential internships provide this opportunity, as students and preceptors can discuss and implement intended learning plans. Previous studies evaluating experiential training as a learning context for cultural competency further support this argument. ${ }^{10,11}$ For these reasons, internships should be the primary setting for competency development and assessment.

A dedicated experiential internship, focused on cultural awareness training and mapped to the AFPC and NAPRA 
competencies given above, ${ }^{4,5}$ is required to ensure that students achieve these educational outcomes. However, there should not necessarily be an assumption that specific internships (international, public health, rural, inner city, etc.) will provide cultural diversity. Instead, a sample of internship sites should be carefully selected and preceptors suitably trained to provide an environment conducive to student reflection and self-growth from culturally sensitive interactions. Then, students and preceptors can work together to develop individualized student objectives specific to cultural awareness, practice recognition of situations of cultural sensitivity, reflect on identified encounters, and assess students' self-awareness and associated performance throughout the internship. The likely keys to success of this model include preceptor training on instruction and assessment of cultural awareness competencies; a focus on self-reflection, self-awareness, and selfassessment; and monitoring of students' achievement of the intended learning outcomes. One might argue that any internship setting will provide adequate opportunities for students to recognize "cultural moments" and will promote self-reflection and awareness. However, without specification of clear objectives and intended learning outcomes, this approach risks overlooking cultural awareness training as a whole.

Outcomes and competencies related to cultural awareness and competency are essential curricular components, yet they are difficult to teach and assess. As no particular interaction or setting can be guaranteed to provide a culturally diverse scenario for each student, experiential internships must be relied on as the most appropriate context for achievement of these competencies. To ensure that educational goals are met, a dedicated mandatory internship with objectives, instruction, and assessments mapped to culture-related outcomes is necessary. By this means, students and preceptors can work together to promote self-reflection and awareness for future success within interactions of cultural diversity.

\section{References}

1. Thomas SB, Fine MJ, Ibrahim SA. Health disparities: the importance of culture and health communication. Am J Public Health. 2004;94(12):2050.

2. Betancourt JR, Green AR, Carrillo JE, Ananeh-Firempong O 2nd. Defining cultural competence: a practical framework for addressing racial/ethnic disparities in health and health care. Public Health Rep. 2003;118(4):293-302.

3. Shaya FT, Gbarayor CM. The case for cultural competence in health professions education. Am J Pharm Educ. 2006;70(6):124.

4. Educational outcomes. Edmonton (AB): Association of Faculties of Pharmacy of Canada; 2010 [cited 2016 Mar 9]. Available from: http:// afpc.info/node/39

5. Professional competencies for Canadian pharmacists at entry to practice. Ottawa (ON): National Association of Pharmacy Regulatory Authorities; 2014 [cited 2016 Mar 9]. Available from: http://napra.ca/content_files/ files/comp_for_cdn_pharmacists_at_entrytopractice_march2014_b.pdf

6. Beach MC, Price EG, Gary TL, Robinson KA, Gozu A, Palacio A, et al. Cultural competence: a systematic review of health care provider educational interventions. Med Care. 2005;43(4):356-73.

7. Campinha-Bacote J. Many faces: addressing diversity in health care. Online J Issues Nurs. 2003;8(1):123-30.

8. Kumas-Tan Z, Beagan B, Loppie C, MacLeod A, Frank B. Measures of cultural competence: examining hidden assumptions. Acad Med. 2007; $82(6): 548-57$
9. Prasad SI, Nair P, Gadhvi K, Barai I, Danish HS, Philip AB. Cultural humility: treating the patient, not the illness. Med Educ Online. 2016;21:30908.

10. Deepti V, Caligiuri FJ. Reinforcing cultural competency concepts during introductory pharmacy practice experiences. Am J Pharm Educ. 2010; 74(4):129.

11. Haack S. Engaging pharmacy students with diverse patient populations to improve cultural competence. Am J Pharm Educ. 2008;72(5):124.

\section{Jayesh Changela \\ Madison Schmidt \\ Carly Caixeiro \\ BSP Candidates 2016 \\ College of Pharmacy and Nutrition \\ University of Saskatchewan \\ Saskatoon, Saskatchewan}

Kyle J Wilby, BSP, ACPR, PharmD

Assistant Professor

College of Pharmacy, Qatar University

Doha, Qatar

Competing interests: None declared.

\section{THE "CON" SIDE}

A direct relationship exists between health practices and culture. Of the factors known to influence health beliefs and behaviours, culture is the most influential. ${ }^{1}$ Cultural values are unique to each person. This diversity is influenced by all or a combination of religious affiliation, language, physical size, gender, sexual orientation, age, disability (physical and mental), political orientation, socioeconomic status, occupational status, and geographic location. ${ }^{1}$ The mixing of these elements contributes to both inter- and intra-cultural differences. To meet the needs of a culturally diverse patient population and to function effectively in a culturally diverse workforce, pharmacists must be culturally competent.

While we acknowledge the necessity of cultural competency training, we disagree with multiple aspects of the mandate implied by the question for this Point Counterpoint exchange. We will explore some of the limitations of the proposition and provide alternative training solutions.

\section{Awareness Is Not Enough}

Cultural awareness is the "process of conducting selfexamination of one's own biases towards other cultures and the in-depth exploration of one's cultural and professional background." ${ }^{2}$ Cultural competence is the "process in which the healthcare professional continually strives to achieve the ability and availability to effectively work within the cultural context of a client (family, individual or community). It is a process of becoming culturally competent, not being culturally competent" 
[emphasis added]. ${ }^{2}$ This model includes cultural awareness, cultural knowledge, cultural skill, cultural encounters, and cultural desire as the pillars of cultural competence. On the basis of this model, we believe that awareness is not enough and that competence should be the benchmark. Pharmacists must not only be aware of their potential biases, but must also gain cultural knowledge, adapt their attitudes and behaviours to practice with cultural sensitivity, engage in multiple cultural encounters, and possess the desire to develop this type of competency.

\section{Training Methods}

Pharmacy schools throughout North America have incorporated cultural competency training into their curricula; however, this has been done in varying depth and breadth, and through different methods. ${ }^{3}$ Some pharmacy programs have implemented lecture series in the didactic portion of their curricula, while others have entered formal memoranda of understanding with international pharmacy schools for experiential internships. ${ }^{4}$ There is limited evidence as to which of these training methods is the most effective. Given the range in patient and provider diversity and comprehensive cultural competency models that we have described, it is unlikely that experiential internships alone will give learners the sustained exposure necessary to attain competency. This type of training typically provides brief exposure to a specific culture. Assessments of training methods have shown that improvements in cultural competency were associated with higher-intensity training for longer durations. ${ }^{5}$

\section{Competency Assessment}

Our literature search did not identify a consistently utilized assessment tool for cultural awareness or competency within pharmacy or the larger health care sector. There are multiple self-perception and attitude assessments, ${ }^{6}$ but no single standard for deeming that a pharmacy student or pharmacist is culturally aware or competent. The absence of assessment standards makes it difficult to evaluate whether learners are achieving training outcomes. This deficiency may also limit the ability to identify culturally competent preceptors.

\section{Impact of Training}

The impact of cultural competency training was evaluated in a systematic review. ${ }^{7}$ The majority of studies included in the review evaluated cultural competency in physicians and nurses (both students and practising professionals), utilizing beforeand-after evaluations, and were conducted in the United States. Multiple teaching methods and content were evaluated; of note, no two studies evaluated the same curricula. Results indicated that training improved health care professionals' knowledge, attitudes, and skills and patients' satisfaction, but there was inconclusive evidence to show an improvement in adherence to therapy, patient outcomes, and equity of services across racial and ethnic groups, and there was minimal data on the cost-effectiveness of training. ${ }^{7}$ One of the 34 studies included pharmacy students who participated in a 5-week interdisciplinary course focusing on African American culture in 1995. The course content included lectures, cases, and cultural clinical experiences. Pre- and post-course self-assessments showed improvement in students' knowledge and attitudes. ${ }^{7}$ The lack of standardized training and evaluation methods limits robust analysis of the impact of training methods. Additionally, the limited evidence indicative of positive patient outcomes and costeffectiveness may deter commitment from stakeholders.

\section{Demand and Capacity}

Recent increases in enrolment in Canadian pharmacy programs and the implementation of entry-level PharmD programs has increased the demand for experiential sites. The current experiential training model cannot keep up with this demand, as hospitals and community sites are increasingly hesitant to further invest in training. ${ }^{8}$ Therefore, it may be challenging to create or identify the additional placement sites, with qualified preceptors, that are needed for experiential internships focusing on cultural awareness. Capacity constraints are more profound in the international setting, as establishing and maintaining these internships takes time and is complex. Financial capacity constraints also exist. Studying away from home, regardless of location, is costly, and many students are reluctant to take on the additional debt. ${ }^{9}$

\section{Alternatives}

Despite the array of opinions, there seems to be some consensus that becoming culturally competent is a process, one that requires ongoing reinforcement and exposure. Therefore, pharmacy programs should incorporate cultural competency training throughout their curricula. The concept of and selfreflection about potential cultural biases against patients and classmates or colleagues should be implemented early in the course of study. Cultural factors should be incorporated into care planning in both simulation and experiential settings. Objective Structured Clinical Examinations (OSCEs) should incorporate a cultural competency station and should utilize culturally diverse standardized patients. Technology today enables international collaboration with other pharmacy programs around the world for teleconferences, e-labs, and e-tutorials. Guest lecturers could be invited to facilitate integrated cases, discussions, or activities. We believe the population in Canada is diverse enough to allow for a range of cultural encounters throughout experiential internships. Perhaps the mandate should be that every experiential internship includes learning objectives that address cultural competency. There is also the option of partnering with employers or organizations that offer experiential internships to provide 
site-specific cultural competency training based on the demographic characteristics of the populations they serve.

\section{Conclusion}

Mandating that at least one experiential internship focusing on cultural awareness training be incorporated into entry-topractice PharmD curricula is not the optimal solution to address the training needs of pharmacy students. There are multiple gaps in training and assessment methods and in supporting evidence for a positive impact on patient outcomes. Furthermore, the current experiential training model cannot keep up with increasing demand. We suggest setting competency (instead of awareness) as the desired training outcome, incorporating cultural competency training throughout all course work, and developing a valid rubric for assessment based on demonstrable behaviours. Viable alternatives to help address capacity limits have been provided.

We have an opportunity to create a unique cultural competency curriculum as entry-to-practice PharmD programs are implemented across Canada. Experiential internships are one key part of that curriculum, but not the only part. Cultural competency education is not an event, it is a process. Thus, training should not be delivered in boluses, but should be continuously infused throughout the entire educational experience.

\section{References}

1. Campinha-Bacote J. Many faces: addressing diversity in health care. Online J Issues Nurs. 2003;8(1):123-30.

2. The process of cultural competence in the delivery of healthcare services. Cincinnati (OH): Transcultural C.A.R.E. Associates; 2015 [cited 2016 Mar 9]. Available from: http://transculturalcare.net/the-process-of-culturalcompetence-in-the-delivery-of-healthcare-services/

3. American College of Clinical Pharmacy, O'Connell M, Rodriguez de Bittner M, Poirier T, Karaoui L, Echeverri M, Chen AM, et al. Cultural competency in health care and its implications for pharmacy part 3A: emphasis on pharmacy education, curriculums, and future directions. Pharmacotherapy. 2013; 33(12):e347-67.

4. Fischer K. All abroad! Overseas study required. Chron Higher Educ. 2008; 54(41):1-6.

5. Sales I, Jonkman L, Connor S, Hall D. A comparison of educational interventions to enhance cultural competency in pharmacy students. Am J Pharm Educ. 2013;77(4):76.
6. Echeverri M, Brookover C, Kennedy K. Assessing pharmacy students' selfperception of cultural competence. J Health Care Poor Underserved. 2013; 24(1 Suppl):64-92.

7. Beach MC, Price EG, Gary TL, Robinson KA, Gozu A, Palacio A, et al. Cultural competence: a systematic review of health care provider educational interventions. Med Care. 2005;43(4):356-73.

8. Hall K, Musing E, Miller DA, Tisdale JE. Experiential training for pharmacy students: time for a new approach. Can J Hosp Pharm. 2012;65(4):285-93.

9. Owen C, Breheny P, Ingram R, Pfeifle W, Cain J, Ryan M. Factors associated with pharmacy student interest in international study. Am J Pharm Educ. 2013; 77(3):54.

\section{Darren Bogle}

Kayla Glatt

Trevor Kwan, BSC

BSP Candidates 2016

College of Pharmacy and Nutrition

University of Saskatchewan

Saskatoon, Saskatchewan

Maria Paiva, BSP, BCPS, PharmD

Clinical Pharmacist

Sidra Medical and Research Center

Adjunct Clinical Lecturer, Clinical Pharmacy and Practice

College of Pharmacy, Qatar University

Doha, Qatar

Competing interests: None declared.

Acknowledgements: The authors wish to thank the local support team in Ghana, especially Samuel Boakye and James Obiri.

The Pro and Con articles for this issue's "Point Counterpoint" column were developed from an elective specialty experiential training internship in Mampong, Ghana, as part of the Structured Experiential Training Program, College of Pharmacy and Nutrition, University of Saskatchewan. 